
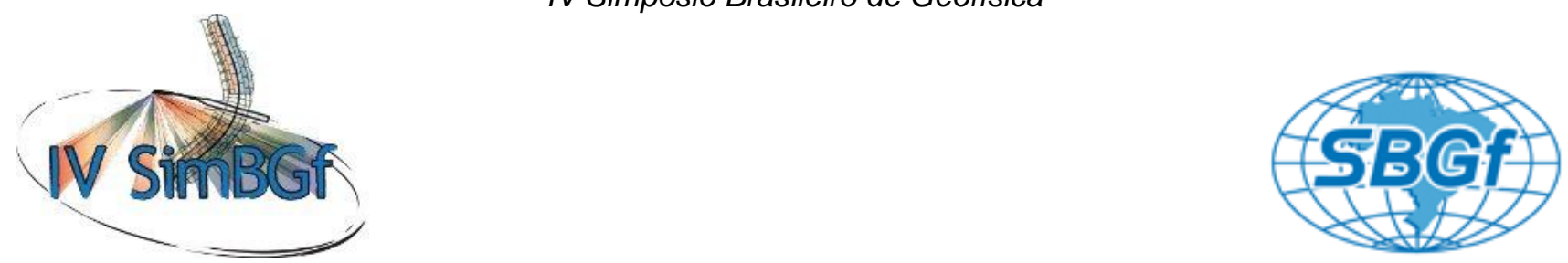

\title{
Determinação da Porosidade com Algoritmo Inteligente
}

Cesar Carneiro; Carolina Barros \& André Andrade - UFPA

Copyright 2010, SBGf - Sociedade Brasileira de Geofísica

Este texto foi preparado para a apresentação no IV Simpósio Brasileiro de Geofísica Brasília, 14 a 17 de novembro de 2010. Seu conteúdo foi revisado pelo Comitê Técnico do IV SimBGf, mas não necessariamente representa a opinião da SBGf ou de seus associados. É proibida a reprodução total ou parcial deste material para propósitos comerciais sem prévia autorização da SBGf.

\section{Resumo}

A porosidade expressa o volume de fluido em uma rocha sedimentar, definindo a sua capacidade de armazenamento de fluido e a sua qualidade como rocha reservatório. A determinação de valores realistas para a porosidade impacta diretamente sobre o cálculo da saturação e, consequentemente, na avaliação das reservas de hidrocarboneto. Um dos métodos mais difundidos para o cálculo da porosidade utiliza a associação dos perfis de densidade e porosidade neutrônica, que produz valores de porosidade corrigidos da argilosidade. Os pontos destes perfis em um gráfico apresentam característicos padrões angulares, que podem ser convenientemente interpretados por um algoritmos inteligente, baseado em uma rede neural artificial, do tipo competitiva angular, que a partir do reconhecimento destes padrões angulares realiza 0 zoneamento do poço, possibilitando o cálculo da porosidade com os valores convenientes das propriedades físicas para cada litologia. A avaliação desta metodologia é realizada com perfis de porosidade e resultados da análise de testemunhos de poços do Campo de Namorado, na Bacia de Campos. Brasil.

\section{Introdução}

A porosidade expressa a fração do volume de uma rocha reservatório que é totalmente preenchida por fluido. $\mathrm{Em}$ termos da caracterização petrofísica de um reservatório, a porosidade é uma das mais importantes propriedades, pois determina a sua capacidade de armazenamento de fluidos e tem impacto direto sobre o cálculo das saturações e, consequentemente, sobre a quantificação das reservas de hidrocarbonetos, que é determinante para o estabelecimento das estratégias de explotação de um poço.

Os métodos tradicionais da avaliação de formação para o cálculo da porosidade utilizam os perfis de porosidade (densidade, sônico e porosidade neutrônica), com base em um modelo de rocha reservatório, segundo o qual as medidas registradas nos perfis de porosidade são influenciadas pelas propriedades físicas de cada constituinte da rocha, proporcionalmente aos seus respectivos volumes relativos. Assim, o cálculo de valores realistas da porosidade requer, necessariamente, informações confiáveis sobre as propriedades físicas mensuradas pelos perfis de porosidade na matriz, no fluido e na argila, que, se não convenientemente consideradas, induzem a obtenção de valores profundamente otimistas para a porosidade.

A matriz é o conjunto de minerais constituintes do arcabouço da rocha reservatório. Para o cálculo da porosidade, a matriz é caracterizada pelo conjunto de propriedades físicas mensuradas pelas ferramentas de porosidade, que, normalmente, são obtidas, quando possível, por meio de medidas diretas sobre amostras de testemunhos. Uma medida comum na análise de testemunho é a densidade do grão, no entanto medidas do tempo de trânsito e da porosidade neutrônica são raras. No caso da ausência de medidas diretas, é comum, na avaliação de formação, a adoção das propriedades do mineral principal para a caracterização da matriz.

Para a argila, a variabilidade na sua composição química resulta em uma grande variação das suas propriedades físicas, de tal modo, que é raro o estabelecimento de valores constantes, a partir do seu principal componente. Normalmente, são raros os testemunhos nas camadas de folhelho e não é pratica comum, a separação da fração argila das rochas reservatório na análise de testemunho. Para o cálculo da porosidade, é comumente assumido o conjunto de valores dos perfis, medidos nas camadas de folhelho adjacentes às camadas reservatório, para 0 estabelecimento das propriedades físicas da argila. Neste caso, prevalece a premissa de que a argila no folhelho é geologicamente semelhante à argila presente na constituição da rocha reservatório.

A caracterização física do fluido no poro, em termos de medidas diretas é bastante complexa, em função da ausência de amostras não contaminada e pelo desconhecimento, a priori, da sua composição, tanto química, quanto em termos dos volumes relativos de água de formação e de hidrocarboneto. Para o cálculo da porosidade é pratica comum, a adoção das propriedades físicas da água doce, para a caracterização do fluido no poro.

O método densidade-neutrônico (Schlumberger, 1987) é um dos mais difundidos métodos para o cálculo da porosidade, tomando a associação dos perfis de densidade e porosidade neutrônica, para produzir uma primeira estimativa para a porosidade corrigida pelo efeito da argilosidade sobre estes perfis. A apresentação no Gráfico densidade-neutrônico, dos pontos formados pelos valores do par de perfis para cada profundidade de uma mesma litologia forma um agrupamento, que pode ser caracterizado como um padrão angular. Um conjunto de pontos no plano apresenta um padrão angular, 
quando estão dispostos, aproximadamente, próximos à direção de um único vetor.

Apresenta-se um algoritmo inteligente, que se responsabiliza pela obtenção de valores realistas para a porosidade, a partir identificação dos padrões angulares no Gráfico densidade-neutrônico, que possibilita o zoneamento do poço, com a determinação da densidade e da porosidade neutrônica da argila e do mineral principal de cada camada reservatório.

Os algoritmos inteligentes formam uma grande classe de técnicas computacionais, como as redes neurais artificiais, a computação evolutiva, e a inferência fuzzy, principalmente utilizados para a realização de análise e interpretação de grandes massas de dados. Os algoritmos inteligentes são ferramentas poderosas aplicados a vários campos da ciência e da engenharia, transformando dados em informação e informação em conhecimento (Nikravesh, 2004).

O método proposto para a interpretação do Gráfico densidade-neutrônico é baseado em uma rede neural artificial, do tipo competitiva, com treinamento não supervisionado, denominada como rede competitiva mínimo angular. Esta rede é uma variante da rede competitiva angular (Barros \& Andrade, 2008), que é especializada para a solução de problemas de identificação de padrões angulares.

A apresentação desta metodologia é realizada através de dados sintéticos que satisfazem a equação geral dos perfis de porosidade e a sua avaliação são utilizados perfis de porosidade convencionais registrados em poços do Campo de Namorado, na Bacia de Campos. Brasil.

\section{Metodologia}

O cálculo da porosidade com o método densidadeneutrônico envolve a solução de um sistema linear, com duas equações, baseadas nas equações dos perfis de densidade e porosidade neutrônica, com duas incógnitas, a porosidade e a argilosidade. O impedimento para uma simples solução numérica é o desconhecimento das constantes, que representam as propriedades físicas da matriz e da argila, nas duas equações, Assim, na prática tem-se um sistema subdeterminado, com duas equações e seis incógnitas, quatro propriedades físicas, densidade e porosidade neutrônica da matriz e da argila, a argilosidade e a porosidade. A sua solução deste sistema ocorre na forma gráfica, com a necessidade de informação a priori sobre a litologia.

O objetivo desta metodologia é produzir estimativas para as densidades e porosidades neutrônicas da matriz e da argila, independente da existência de quaisquer informações a priori, como ocorre na maioria dos casos de avaliação de formação de poços não testemunhados.

\section{Método Densidade-Neutrônico}

O método densidade-neutrônico (Schlumberger, 1987) é um dos mais difundidos métodos, que utilizam a associação de dois perfis de porosidade,no caso, o perfil de densidade e o perfil de porosidade neutrônica para o cálculo simultâneo da porosidade e da argilosidade.

Tomando-se a equação geral dos perfis de porosidade escrita para cada perfil, na forma de um sistema de equações lineares,

$$
\left\{\begin{array}{l}
\rho_{b}=\phi \rho_{w}+V_{s h} \rho_{s h}-\left(1-\phi-V_{s h}\right) \rho_{m} \\
\phi_{n}=\phi \phi_{w}+V_{s h} \phi_{s h}-\left(1-\phi-V_{s h}\right) \phi_{m}
\end{array}\right.
$$

$\mathrm{Na}$ equação $1, \rho_{b}$ é o valor registrado no perfil de densidade; $\phi_{n}$ é o valor registrado no perfil de porosidade neutrônica; $\phi$ é a porosidade, corrigida pelo efeito da argilosidade $\left(V_{s h}\right)$. Os termos, $\rho_{m}, \rho_{s h}$ e $\rho_{w}$ representam as densidades da matriz, da argila e da água. Os termos, $\phi_{m}, \phi_{s h}$ e $\phi_{w}$ representam as porosidades neutrônicas da matriz, da argila e da água.

A solução clássica para a porosidade é dada por

$$
\phi=\frac{\phi_{D} \phi_{N_{s h}}-\phi_{N} \phi_{D_{s h}}}{\phi_{N_{s h}}-\phi_{D_{s h}}}
$$

Na equação 2, os termos $\phi_{D}$ e $\phi_{N}$ são os valores de porosidade calculados pelos perfis de densidade $\mathrm{e}$ porosidade neutrônica, considerando a rocha limpa $\left(V_{s h}=0\right)$, na forma,

$$
\phi_{D}=\frac{\rho_{m}-\rho_{b}}{\rho_{m}-\rho_{w}} \text { e } \phi_{N}=\frac{\phi_{m}-\phi_{n}}{\phi_{m}-\phi_{w}}
$$

Os termos $\phi_{D_{s h}}$ e $\phi_{N_{s h}}$ são os valores de porosidade calculados pelos perfis de densidade e porosidade neutrônica para a argila, na forma,

$$
\phi_{D_{s h}}=\frac{\rho_{m}-\rho_{s h}}{\rho_{m}-\rho_{w}} \text { e } \phi_{N_{s h}}=\frac{\phi_{m}-\phi_{s h}}{\phi_{m}-\phi_{w}}
$$

A Figura 1 mostra a forma de solução gráfica do sistema linear apresentado na equação 1, através do Gráfico densidade-neutrônico.

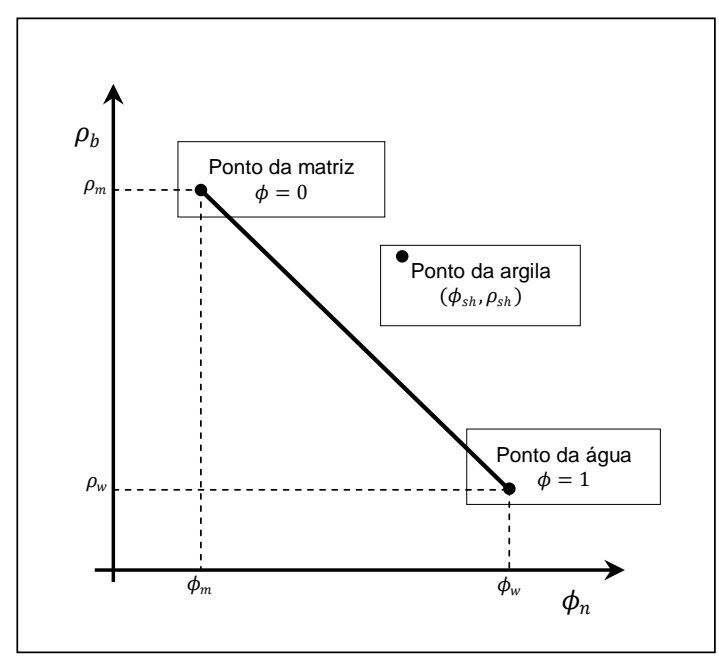

Figura 1 - Gráfico densidade-neutrônico. 
Considerando a água doce como fluido intersticial, pode-se traçar retas de porosidade unindo o ponto da água ao ponto de matriz para cada mineral principal na constituição as rochas reservatório. O ponto da argila é determinado pelos pares ordenados formados pelos perfis de densidade e porosidade neutrônica registrados frentes aos folhelhos adjacentes às camadas reservatório. Pontos de litologia limpa alinham-se nas proximidades da reta construída a partir do seu mineral principal, distribuindo-se de acordo com a sua porosidade. Pontos de litologia suja são deslocados na direção do ponto da argila.

\section{Redes Neurais Artificiais}

Sob a denominação geral de algoritmos inteligentes encontra-se um grande grupo de métodos computacionais, como os sistemas especialistas, a inferência fuzzy, as redes neurais artificiais e os algoritmos evolutivos, que inserem flexibilidade e capacidade de processamento da informação para solução de problemas da vida real. A principal característica dos algoritmos inteligentes é a sua capacidade de operar e encontrar uma solução aceitável para um dado problema, em condições severas de imprecisão, incerteza e parcialidade da informação, além de apresentar uma grade tolerabilidade a falhas e robustez, principalmente para o caso da solução de problemas de decisão. Em outras palavras, os algoritmos inteligentes apresentam uma oportunidade para a solução de problemas que envolvem a ambiguidade do pensamento e as incertezas da vida real, (Nikravesh, 2004).

As redes neurais artificiais são técnicas computacionais cujas propriedades são inspiradas em um modelo simplificado do funcionamento do cérebro dos mamíferos, cuja principal característica é a aquisição de conhecimento através da experiência. Assim, as redes neurais artificiais podem extrair, armazenar e utilizar a informação experimental, o que as difere completamente de um algoritmo seqüencial, uma vez que a informação em uma rede neural não é armazenada em um local predefinido, mas sim distribuída por toda a sua arquitetura.

Uma rede neural é composta por um conjunto de elementos processadores não lineares, denominados neurônios, que são dispostos em um arranjo fixo, ou camada. As diversas camadas são interligadas por um conjunto de pesos sinápticos. Toda esta estrutura é conhecida como arquitetura. A forma como a informação propaga-se através das camadas e a arquitetura define as características da rede neural.

O aspecto das redes neurais, aqui explorado é o da capacidade de reconhecimento de padrões, ou de extração de características comuns e estatisticamente relevantes presentes nos dados de entrada. Uma rede neural com estas características é conhecida como rede neural competitiva. A sua arquitetura mais comum é composta por duas camadas: a camada de entrada e a camada competitiva. Os neurônios competitivos são forçados a competir entre si, de modo que apenas um neurônio (neurônio vencedor) esteja ativo ou produza um sinal de saída não-nulo a cada apresentação de um dado de entrada.

Uma rede neural competitiva é caracterizada por um treinamento não supervisionado, onde a partir de um conjunto de critérios é estabelecida a forma de atualização dos pesos sinápticos, que determina a existência de um único neurônio vencedor na camada competitiva. Esta característica busca possibilitar a extração de características estatisticamente relevantes presentes nos dados de entrada. Neste tipo de rede os dados de entrada são tratados de forma a explicitar propriedades comuns presentes em seu conjunto. Diferente de outra arquitetura de rede neural artificial, o resultado útil de uma rede competitiva pode ser a posição na camada competitiva ou o conjunto de pesos do neurônio de vencedor.

\section{Rede Competitiva Mínimo Angular}

A rede competitiva mínimo angular é derivada da arquitetura e das estratégias de treinamento e operação apresentados pela Rede Competitiva Angular (Barros \& Andrade, 2008). A rede competitiva angular é um particular tipo de rede competitiva desenvolvida para realizar a extração de possíveis relações angulares por ventura existentes nos dados de entrada. O caso típico de relação angular é denominado como padrão angular, definido como uma particular disposição apresentada pelos dados de entrada, quando formam agrupamentos, aproximadamente, próximos à direção de um vetor. A identificação destes particulares vetores possibilita a classificação dos agrupamentos.

No caso bidimensional, cada dado de entrada é um ponto do plano ao qual está associado um vetor posição, transformado para o seu vetor unitário, que é representado na forma de um número complexo. Assim, as operações vetoriais são substituídas pelas operações aritméticas dos números complexos.

A arquitetura da rede competitiva mínimo angular é mostrada na Figura 2.

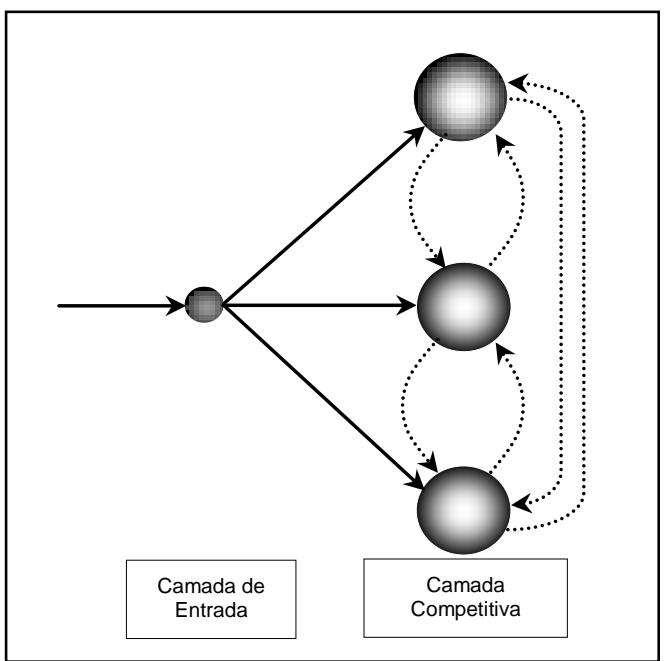

Figura 2 - Arquitetura da rede competitiva mínimo angular. 
A rede competitiva mínimo angular utiliza a estratégia de operação da rede competitiva angular buscando classificar pontos no plano em relação as classes representadas por vetores de referência. Um ponto, representado por seu vetor posição, é classificado em uma classe representada pelo vetor de referência (vetor posição) que forma o menor ângulo entre eles.

Cada neurônio competitivo produz como sinal de saída, o resultado da avaliação da sua função de ativação para potencial de entrada de cada elemento do vetor de entrada.

O potencial de entrada $\left(u_{j}\right)$ é definido na forma

$$
u_{j}=\operatorname{real}\left(x_{i} \bar{w}_{j}\right)=\cos (\theta)
$$

$\mathrm{Na}$ equação 5 , o potencial de entrada é o produto interno de dois vetores, que cosseno do ângulo entre eles, escrito na forma complexa. O termo $x_{i}$ é um elemento do vetor de entrada, escrito como um número complexo. Neste caso, o plano cartesiano é interpretado como o plano complexo. O termo $\bar{w}_{j}$ é o conjugado complexo de um elemento do vetor de pesos sinápticos. $O$ vetor de pesos sinápticos é formado pelos vetores de referência, escritos na forma complexa, que representam as possíveis classes.

A função de ativação de cada neurônio competitivo determina o seu sinal de saída $\left(y_{j}\right)$. Para efeito de classificação, a função de ativação é tomada como uma função afim do potencial de entrada, como mostrado na Figura 3.

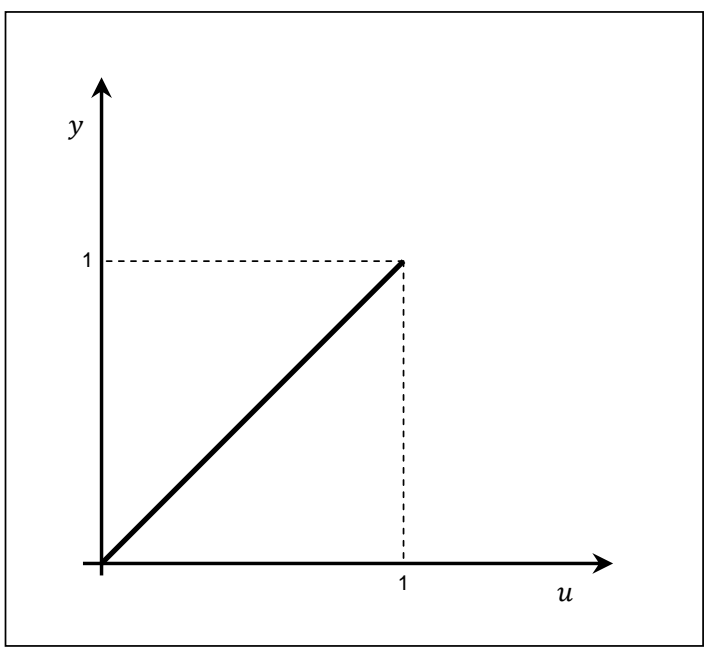

Figura 3 - Função de ativação.

A competição entre os neurônios é governada pelas regras de competição. Para a realização de uma classificação com base na proximidade entre pontos, a regra de competição estabelece como neurônio vencedor, aquele com o maior valor de sinal de saída.

Algoritmo Inteligente

O Gráfico densidade-neutrônico é construído considerando apenas três matrizes de rocha reservatório, com os minerais principais: quartzo para o arenito, calcita para o calcário e dolomita. As propriedades físicas destes minerais formam os pontos de matriz, como mostrado na Tabela 1.

Tabela 1 - Pontos de matriz.

\begin{tabular}{ccc}
\hline Mineral & $\rho_{b}$ & $\phi_{n}$ \\
\hline Quartzo & 2,65 & $-0,05$ \\
Calcita & 2,71 & 0 \\
Dolomita & 2,87 & 0,05 \\
\hline
\end{tabular}

As direções das retas de porosidade determinam as direções dos vetores de referência, tomados como os vetores unitários dos vetores formados pela subtração do vetor posição do ponto de cada matriz pelo vetor posição do ponto da água.

Um ponto do perfil, no Gráfico densidadeneutrônico, é representado pelo par ordenado $\left(\phi_{n}, \rho_{b}\right)$ formado pelos valores dos perfis de porosidade neutrônica e densidade para uma mesma profundidade. A partir dos pontos do perfil é construído o vetor complexo de entrada, que é formado pelos vetores unitários da subtração do vetor posição de um ponto do perfil pelo vetor posição do ponto da água.

O algoritmo inteligente para a interpretação do Gráfico densidade-neutrônico é composto por duas redes competitiva mínimo angular e opera em dois passos.

A primeira atividade é a determinação de um vetor de referência para representar a argila. Uma rede competitiva mínimo angular é construída com um neurônio de entrada e três neurônios competitivos, representando cada uma das matrizes. A operação da rede competitiva mínimo angular realiza uma primeira classificação dos pontos do perfil. Para a definição do ponto de argila são considerados apenas os pontos do perfil classificados como dolomita. O ponto da argila é tomado como o ponto do perfil correspondente ao elemento do vetor de entrada mais afastado do vetor de referência que representa a dolomita. $O$ vetor correspondente ao ponto de argila é transformado em vetor de referência para a segunda rede.

Para o cálculo da porosidade esta classificação não é satisfatória por não produzir o zoneamento do poço, em termos das matrizes de cada camada.

Uma segunda rede competitiva mínimo angular é construída com a mesma arquitetura da primeira, mas com o potencial de entrada escrito na forma,

$$
u_{j}=\operatorname{real}\left(x_{i} \alpha \bar{w}_{j}\right)=\cos (\theta)
$$

O parâmetro $\alpha$, na equação 6 , busca 0 prevalecimento da continuidade geológica em profundidade. Assim, ele altera a possibilidade de um neurônio vencer a competição por um ponto do perfil, em função do número de vezes em que ele venceria a competição para outros pontos situados na vizinhança em profundidade deste ponto. 


\section{Resultados}

Considera-se um caso com dados sintético de um intervalo de um poço testemunhado, que atravessa uma seqüência de camadas, com o folhelho, no topo e seguida pelo arenito, calcário e dolomita, na base. A Figura 4 mostra os perfis de raios gama natural, o perfil de densidade, o perfil de porosidade neutrônica. Para efeito de avaliação do algoritmo inteligente, a Figura 4 mostra o zoneamento do poço e o perfil de porosidade adotados para o modelamento dos perfis sintéticos.

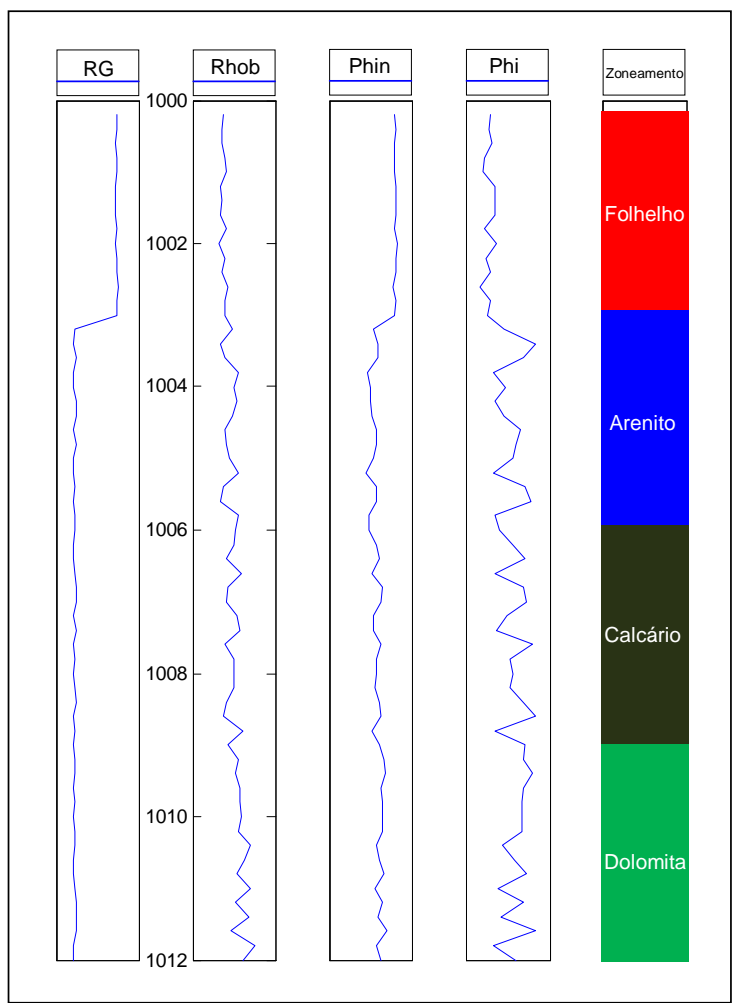

Figura 4 - Dados sintéticos.

A Figura 5 mostra o Gráfico densidade-neutrônico interpretado pelo algoritmo inteligente. São apresentadas as retas de porosidade para cada uma das matrizes consideradas. A escala de cores utilizada para representar cada reta é a mesma adotada para o zoneamento. As cruzes representam os pontos do perfil e a sua cor mostra a classificação realizada pela interpretação do algoritmo inteligente. A estrela em preto mostra o par ordenado $\left(\phi_{s h}, \rho_{s h}\right)$ utilizados na equação 4.

A Figura 6 mostra os resultados para o zoneamento e cálculo da porosidade. A Figura 6-A mostra a comparação entre a porosidade do modelo e a porosidade calculada. A Figura 6-B mostra a comparação com a porosidade calculada sem considerar 0 zoneamento e as propriedades adequadas para as matrizes.

Os resultados da aplicação do algoritmo inteligente para a interpretação do Gráfico densidade-neutrônico com perfis reais são apresentados nas Figuras 7, 8 e 9.

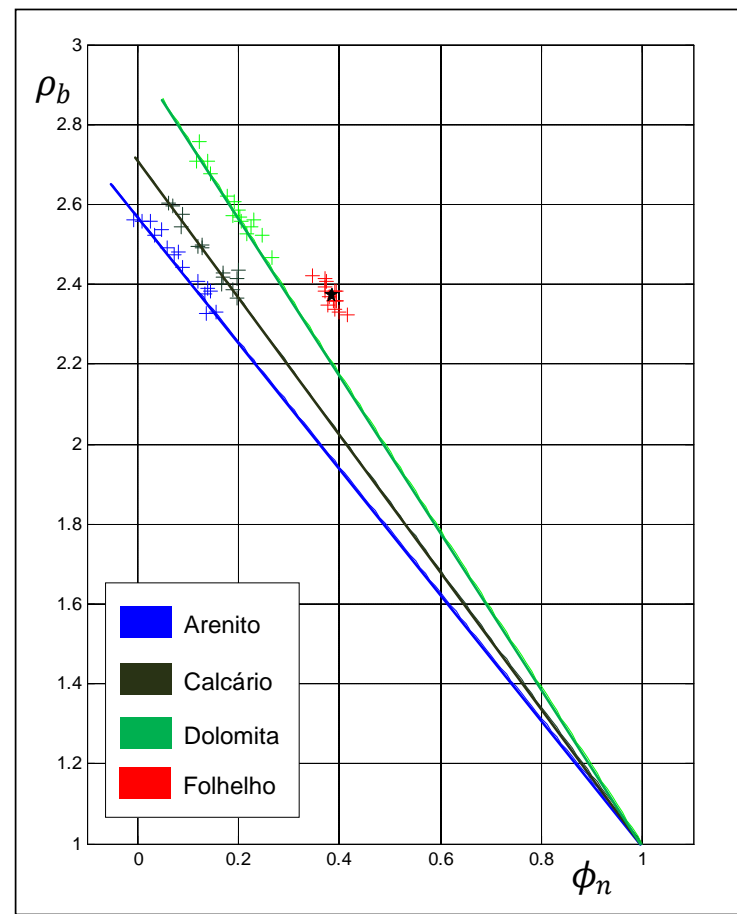

Figura 5 - Dados sintéticos. Gráfico densidadeneutrônico interpretado pelo algoritmo inteligente.

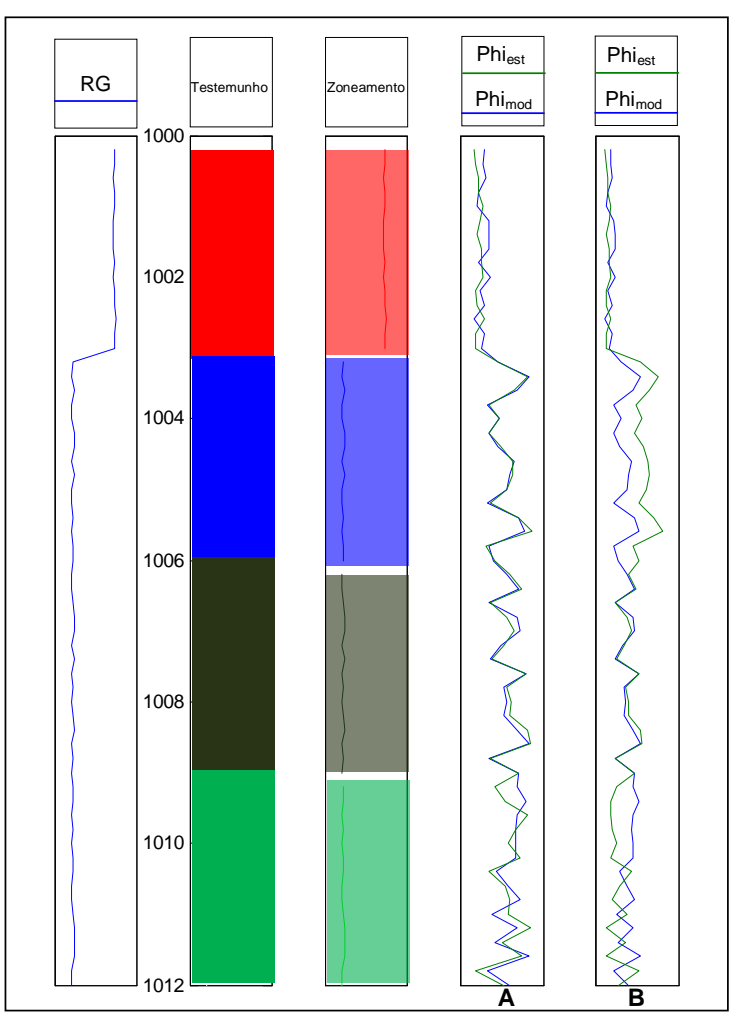

Figura 6 - Dados sintéticos. Resultados do zoneamento e cálculo da porosidade. 


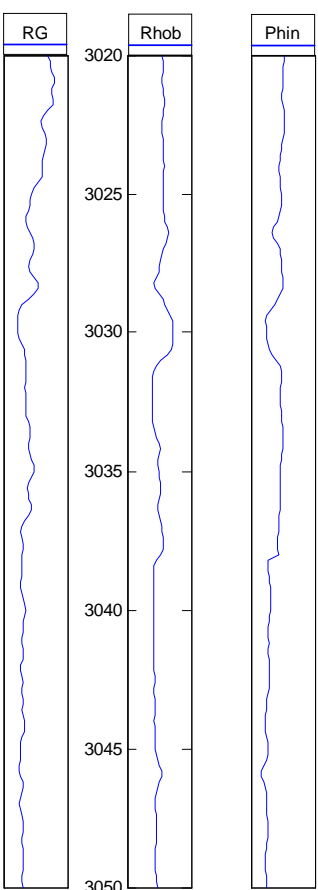

Figura 7 - Perfis reais. Poço do Campo de Namorado. Bacia de Campos.

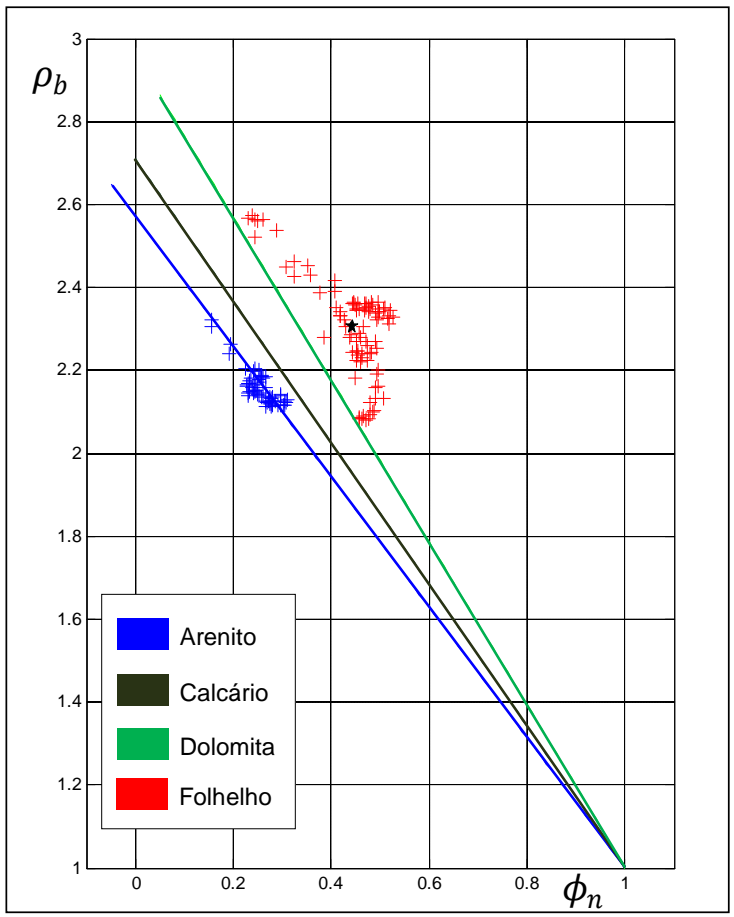

Figura 8 - Dados reais. Gráfico densidadeneutrônico interpretado pelo algoritmo inteligente. A classificação está de acordo com a descrição dos testemunhos.

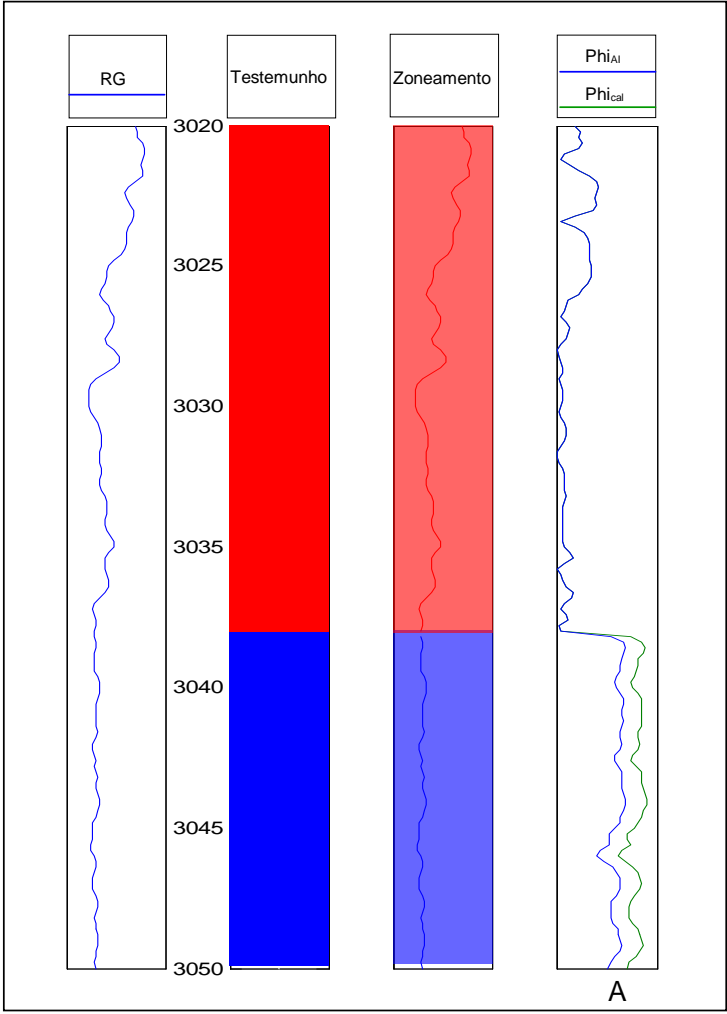

Figura 9 - Dados reais. Zoneamento e cálculo da porosidade realizado pelo algoritmo inteligente. Figura 9-A - Perfis de porosidade.

A Figura 9-A mostra o perfil de porosidade calculado pelo algoritmo inteligente em azul e em verde a porosidade calculada, com a interpretação visual e ausência de informação sobre a matriz.

\section{Conclusões}

O algoritmo inteligente mostrou-se apto para interpretar o Gráfico densidade-neutrônico e produzir valores realistas para a porosidade.

\section{Agradecimentos}

Os autores agradecem pelo apoio a este trabalho a PETROBRAS (Rede de Geofísica) e ao Convenio MCT/UFPA/ANP/PRH-06.

\section{Referências}

Barros, C. \& Andrade, A., 2008. Determination of Water Saturation by Intelligent Algorithm. III SimBGf.

Nikravesh, M., 2004. Soft computed based computational intelligent for reservoir characterization, Expert Systems with Applications. V: 26, 19-38.

Schlumberger Ltda - Log Interpretation Principles/Applications. 1987 